\title{
Rhinocerebral zygomycosis treated with amphotericin B
}

\author{
S. P. Hanley \\ M.R.C.P
}

Brompton Hospital, Fulham Road, London SW3 $6 H P$

\begin{abstract}
Summary
Rhinocerebral zygomycosis is a rare, often fatal opportunistic fungal infection involving the cranial tissues. A diabetic patient with normal humoral and cellular immunity who was successfully treated with amphotericin $B$ and surgery is reported.
\end{abstract}

\section{Case report}

A 32-year-old Phillipino, resident in the U.K. for 9 months, presented with a history of 2 months' anorexia, weight loss, and cough productive of green sputum.

She was found to have diabetic ketoacidosis (pH 6.96, blood glucose $21.5 \mathrm{mmol} / \mathrm{l}$ ) and pulmonary shadowing with cavitation in the left lower lobe diagnosed as tuberculosis. Her sputum was positive for alcohol and acid-fast bacilli using fluorescent staining. Her diabetes was controlled with soluble insulin and intravenous fluids and her tuberculosis treated with rifampicin, ethambutol and isoniazid. She was also treated with penicillin and gentamicin for 2 weeks for purulent sputum.

She gradually improved except for a transient right seventh nerve palsy and pyrexia lasting 7 days. On the tenth day she complained of right maxillary pain and swelling and sinus radiographs showed a fluid level in the right maxillary antrum with erosion of the lateral wall.

On the eleventh day a one-inch ulcer which extended from the midline of the hard palate to involve the alveolar margin was seen. The base was formed by necrotic hard palate perforating through to the right maxillary antrum; there was little inflammation of the ulcer edges. Biopsy of the ulcer demonstrated invasive fungus of the class Zygomycetes and a diagnosis of rhinocerebral zygomycosis was made; mycological cultures of the biopsy and a later excision specimen on Sabouraud's, and Heart Brain Infusion media were unsuccessful. The patient was treated with amphotericin B for 24 days at $1 \mathrm{mg} / \mathrm{kg}$ body-weight and then on alternate days for a further 24 days: a total dose of approximately $1450 \mathrm{mg}$ therapy being given. She was also treated with nystatin and phenol with potassium chlorate mouthwashes.

Her ulcer remained unchanged on this therapy; the necrotic hard palate and alveolar margin were excised on day 69 of treatment. Following this, she has remained well.

\section{Discussion}

A rare complication of diabetes is here reported to emphasize the importance of prompt biopsy and histological examination for fungus in oral ulceration and the prompt instigation of treatment (Abramson, Wilson and Arky, 1967).

Rhinocerebral zygomycosis is an opportunistic fungal infection of which approximately $75 \%$ occur in diabetes mellitus (Chick, Balows and Furcolow, 1975). This disease is extremely rare in the U.K. The first reported case was in 1963 and was of similar presentation with necrosis and perforation of the hard palate in a British resident (LaTouche, Sutherland and Telling, 1963).

In view of the rarity of the disease in the U.K., it is possible that the fungus was already present in the patient's nasopharynx before her arrival in this country. The zygomycetes are, however, widespread saprophytes in the U.K.

Diagnosis depends on the clinical presentation and the histological identification of the fungus with invasion; the differential diagnosis includes fulminant bacterial, Aspergillus and Candida infections.

The reason that this fungus becomes invasive is not clear. Explanations have included the presence of an anti-fungal component in the blood of normal persons absent in the affected subjects, the presence of hyperglycaemia with or without ketoacidosis, or an alteration in immunological status (Chick et al., 1975). It is possible that the use of broad-spectrum antibiotics as used in this case may encourage the risk of fungal infection. This view was not, however, supported by Kligman (1952).

No humoral or cell-mediated abnormality was demonstrated in this patient after the acute phase; the Mantoux test was strongly positive at 1: 10000 . The immunoglobulins were normal with a positive response to challenge to tetanus and TABC vaccines. A positive serological response to the genus Absidia of the class Zygomycetes was obtained 17 days after her admission and reverted to negative by day 69. Serological tests for the genera Mucor and 
Rhizopus were negative as were tests for circulating antigen. Her Candida precipitins were positive and Aspergillus precipitins negative.

No studies of B or $\mathrm{T}$ cell function were performed in the acute phase. However, depressed phytohaemagglutinin response and abnormal PMN chemotaxis have been noted in poorly controlled patients, the latter correlating variably with hyperglycaemia or ketoacidosis (Munro, 1976). Various therapies for this disease have been tried; amphotericin B in conjunction with rigid diabetic control and surgery appears to be the most successful combination.

\section{Acknowledgments}

I gratefully acknowledge the assistance of $\mathrm{Dr} \mathrm{H}$. Nicholson, F.R.C.P., Chest Physician to the Brompton Hospital, Mr
H. J. Shaw, F.R.C.S., Consultant ENT Surgeon to the Royal Marsden Hospital, and Dr D. W. R. Mackenzie, Ph.D., London School of Hygiene and Tropical Medicine.

\section{References}

Abramson, E., Wilson, D. \& ARKy, R.A. (1967) Rhinocerebral phycomycosis in association with diabetic ketoacidosis. Annals of Internal Medicine, 66, 735.

Chick, E.W., Balows, A. \& Furcolow, M.L. (Eds) (1975) Opportunistic Fungal Infections. Proceedings of the Sceond International Conference, 1st edn, pp. 204-214. Charles C. Thomas, Springfield, Ohio.

Kligman, A.M. (1952) Are fungus infections increasing as a result of antibiotic therapy? Journal of the American Medical Association, 149, 979.

LaTouche, C.J., Sutherland, T.W. \& Telling, Maxwell (1963) Rhinocerebral mucormycosis. Lancet, ii, 811.

MUNRo, J.F. (1976) Diabetes and infection. Journal of Antimicrobial Chemotherapy, 2, 322. 